\title{
Antitumor activity of Endostar combined with radiation against human nasopharyngeal carcinoma in mouse xenograft models
}

\author{
JUYING ZHOU $^{1 *}$, LILI WANG ${ }^{1 *}$, XIAOTING XU ${ }^{1}$, YU TU $^{2}$, SONGBING QIN ${ }^{1}$ and YUZHEN YIN ${ }^{1}$ \\ ${ }^{1}$ Department of Radiation Oncology, The First Affiliated Hospital of Suzhou University; \\ ${ }^{2}$ School of Radiation Medicine and Public Health, Medical College of Soochow University, Suzhou, Jiangsu, P.R. China
}

Received April 23, 2012; Accepted July 25, 2012

DOI: $10.3892 / \mathrm{ol} .2012 .856$

\begin{abstract}
Radiation treatment for nasopharyngeal carcinoma (NPC) is common and effective. However, local recurrence occurs frequently. Endostar, a novel recombinant human endostatin, is an antiangiogenic drug with a potent antitumor effect. The present study aimed to observe and explore the radiosensitization effects of Endostar on NPC and its underlying mechanism. The NPC subcutaneous transplantation tumor animal model was established to evaluate the antitumor activity of Endostar combined with radiation (Endostar + radiation) treatment compared with monotherapy (Endostar or radiation). Tumor growth and tumor weight were measured to evaluate the antitumor effect. The level of vascular endothelial growth factor (VEGF) and microvessel density (MVD) were measured using immunohistochemical staining of the tumor tissues. Significant antitumor activity was found in the Endostar + radiation group. The tumor inhibition rates of Endostar, radiation and Endostar + radiation were $27.12,60.45$ and $86.11 \%$, respectively. The VEGF levels in the tumor tissue in the Endostar + radiation group were lower than those in the radiation and control groups. The MVD in the tumor tissues in the Endostar + radiation group was $12.2 \pm 2.5$, lower than that in the Endostar (29.3 \pm 3.4$)$, radiation $(23.5 \pm 3.6)$ and control $(44.7 \pm 5.1)$ groups. These results suggest that Endostar increases the radiation sensitivity of NPC-transplanted tumors in nude mice by lowering VEGF expression. In this study, the NPC animal model was established, which reflects the efficacy of clinical combination
\end{abstract}

Correspondence to: Professor $\mathrm{Yu} \mathrm{Tu}$, School of Radiation Medicine and Public Health, Medical College of Soochow University, 199 Renai Road, Suzhou Industrial Park, Suzhou, Jiangsu 215123, P.R. China

E-mail: tuyu@suda.edu.cn

*Contributed equally

Abbreviations: NPC, nasopharyngeal carcinoma; VEGF, vascular endothelial growth factor; MVD, microvessel density

Key words: Endostar, radiation, antitumor activity, combination therapy, nasopharyngeal carcinoma therapies and the combination of Endostar and radiation. The mechanisms of the combination therapies should be further investigated using this model.

\section{Introduction}

Nasopharyngeal carcinoma (NPC) is one of the most common malignant tumors in China. Presently, the main treatment for NPC is radiation therapy. Over the past 20 years, with the development of imaging, radiation techniques and equipment for NPC, the local control rate among patients in the early stage of the disease has risen to $70-90 \%$. However, in patients in the late stages of the disease (stages III-IV), the local control rate is only $50 \%$, with a five-year overall survival rate of $40-70 \%$ (1). The main causes of treatment failure are local recurrence and distant metastases, which are closely related to each other (2). The key factor that results in the local recurrence of tumors is the presence of tumor cells possessing a resistance to radiation. Therefore, decreasing radiation resistance and increasing radiation sensitivity of tumor cells are of significant clinical relevance.

Previous studies $(3,4)$ have indicated that radiation therapy results in the elevated expression of vascular endothelial growth factor (VEGF), which may contribute to the resistance to radiation in tumors. Therefore, radiation therapy combined with antiangiogenic therapy may be effective in decreasing radiation resistance.

A number of studies have indicated that neovascularization is closely related to the progression and metastasis of tumors (5). The growth of tumors depends on the nutrients and oxygen transported by neovascular vessels. Hence, research has taken a new direction by inhibiting the neovascular formation of tumors and consequently starving the tumor to death. This is known as antiangiogenic therapy. Thus far, endostatin is the endogenous protein which most strongly inhibits the endothelial cells involved in vascular formation. Endostatin inhibits growth, invasion and metastasis by inhibiting tumor angiogenesis without inducing resistance following repeated applications. It also possesses very low toxicity, minimal side effects and is effective in both early- and late-stage tumors (6). However, endostatin is unstable in vitro, difficult to produce and expensive, which limits its clinical application. Endostar is a new recombinant human endostatin synthesized in China and approved by the State Food and Drug Administration in 2005 
for the treatment of non-small cell lung cancer. Endostatin overcomes the limitations of previous recombinant proteins in their clinical applications such as unstable physiochemical characteristics, low water solubility and complicated purification. Therefore, research on the large-scale clinical application of Endostar is possible. Ling et al showed that Endostar exerts its antiangiogenic effects by blocking the VEGF-induced tyrosine phosphorylation of KDR/Flk-1 (7). The study suggested that tumor treatment by radiation combined with Endostar is more effective than radiation alone.

In the present study, the antitumor effects of the combination of Endostar and radiation on subcutaneous transplantation NPC tumor models in BALB/c nude mice were measured, and the microvessel density (MVD) and VEGF levels were also measured to explore the mechanism of the radiosensitization effect of Endostar on NPC.

\section{Materials and methods}

Animals.Four-week-old female BALB-nu/nu(CAnN.Cg-Foxn1 $<$ nu $>/ \mathrm{CrlCrlj} \mathrm{nu/nu)} \mathrm{mice} \mathrm{(weighing} 16 \pm 2 \mathrm{~g}$ ) were obtained from the Shanghai Laboratory Animal Center, Chinese Academy of Sciences (Shanghai, China), and acclimated for 2 weeks in the animal facility before use. The number of animals per experimental group was 6 , as specified in the table and figures. All animal experiments were conducted according to the institution's guidelines for the care and use of laboratory animals.

Tumor cells. The human NPC cell line CNE1, provided by the Shanghai Institute of Biochemistry and Cell Biology, Chinese Academy of Sciences, was cultured in RPMI-1640 medium containing $10 \%$ fetal calf serum (Gibco, Carlsbad, CA, USA) and incubated at $37^{\circ} \mathrm{C}$ under a $5 \% \mathrm{CO}_{2}$ atmosphere.

Human NPC xenograft model. An NPC cell suspension (0.2 ml containing $5 \times 10^{6} \mathrm{CNE} 1$ cells in the log phase) was inoculated subcutaneously into the right lower limb of female BALB/c $\mathrm{nu} / \mathrm{nu}$ mice. The tumor volume reached $391.270 \pm 78.787 \mathrm{~mm}^{3}$ 21 days after inoculation and the mice were randomly divided into 4 groups (A, B, C and D), with 6 mice per group, and the different treatments were initiated (day 1 ).

$B A L B-n u / n u$ mice treatment. Group A received $0.2 \mathrm{ml}$ of normal saline once daily around the tumor for 10 days. Group B was injected with $20 \mathrm{mg} / \mathrm{kg}$ Endostar (Sincere Pharmaceutical Company, Nanjing, China) once daily around the tumor for 10 days. Group $\mathrm{C}$ received $0.2 \mathrm{ml}$ of normal saline once daily around the tumor for 10 days and then $1 \mathrm{~h}$ after administration on day 10,20 Gy of X-rays (5 MeV) was applied to irradiate the NPC tumor once locally (the radiation field was $2 \times 3 \mathrm{~cm}$ and the non-radiation field was protected by lead bricks). Group D was injected with $20 \mathrm{mg} / \mathrm{kg}$ Endostar once daily around the tumor for 10 days and then administered the same radical treatment as was received by group $\mathrm{C}$. Tumor volume, estimated using the equation $\mathrm{V}=$ length $\mathrm{x}$ width ${ }^{2} \mathrm{x} 0.5$, was measured every 4 days after treatment. The percentage of tumor growth inhibition (TGI) was calculated as follows: TGI $(\%)=[1-($ mean change in the tumor volume in each group treated with antitumor drugs / mean change in the tumor volume in the control group)] $x$ 100. The tumor doubling time (DT) was calculated as follows: DT $=\mathrm{d} \times \lg ^{2} /\left(\operatorname{lgV}_{\mathrm{d}}-\lg \mathrm{V}_{0}\right)$, where $\mathrm{d}$ is the length of time between two measurements, $V_{d}$ is the volume of the tumor treated with an antidrug and $\mathrm{V}_{0}$ is the volume of the tumor before the treatment. The mice were euthanized 28 days after treatment and the tumor tissues were collected, weighed and fixed with $4 \%$ formaldehyde.

Immunohistochemistry. Immunohistochemistry was performed using standard streptavidin-biotin complex/ 3,3'-diaminobenzidine (DAB) staining on 4- $\mu$ m thick sections from the paraffin-embedded, formalin-fixed tissue, with DAB acting as a chromogen. The mouse anti-human VEGF antibodies and the rabbit anti-human CD34 antibodies (Boster Biological Technology, Ltd., Wuhan, China) were used to identify the VEGF expression levels and microvessels.

Microvessel density (MVD). The MVD was determined by modification of the methods reported by Mäkitie et al (8). The microvessels were counted from the 5 individual, most highly vascularized areas ('hotspots') containing a high number of CD34 staining sites. The 5 hotspots were selected randomly by scanning an entire immunostained tumor section at x100 magnification. The microvessels were subsequently counted at x400 magnification in each hotspot. Any immunolabeled single or multiple endothelial cells, clearly separate from adjacent tumor cells or interstitial cells, were counted as one microvessel. The MVD was expressed as the mean value of the number of microvessels in the 5 hotspots.

Levels of VEGF in tumors. The levels of VEGF were determined via the semi-quantitative method (9). Three replicates were selected randomly at $\times 200$ magnification and the proportion of the VEGF-positive cells and stain depth were scored in each replicate. The semi-quantitative score was expressed as the product $(\mathrm{S})$ of the score for the proportion of stained cells (S1) and the score for the stain depth (S2), i.e. $\mathrm{S}=\mathrm{S} 1 \mathrm{xS} 2$. The detailed criteria for the scores are shown in Table I. The VEGF expression was designated as negative, weakly positive, positive and strongly positive, respectively, according to the value of the product $(\mathrm{S} \leq 1,1<\mathrm{S} \leq 2,2<\mathrm{S} \leq 4$ and $\mathrm{S}>4)$. The levels of VEGF were expressed as the product of the 2 scores in the 3 replicates.

Statistical analysis. All values are expressed as the mean \pm SD. The differences in the multiple groups were analyzed using one-way analysis of variance (ANOVA). The changes in the inhibition rates of the tumors between groups were compared using the Chi-square test or Fisher's exact test (two-tailed test, $\alpha=0.05)$. All statistical analyses were performed using SPSS 15.0 (SPSS, Inc., Chicago, IL, USA). P<0.05 was considered to indicate a statistically significant result.

\section{Results}

Antitumor activity of Endostar in combination with radiation. The antitumor activity was evaluated on day 39 (38 days after the treatment was initiated). Statistically significant differences were found in tumor weight (Fig. 1A), tumor growth 
Table I. Criteria scores and expressions of VEGF.

\begin{tabular}{|c|c|c|c|c|c|}
\hline $\mathrm{N}$ & $\mathrm{S} 1$ & Stain depth & $\mathrm{S} 2$ & $\mathrm{~S}^{\mathrm{a}}$ & Expression \\
\hline $\mathrm{N}=0$ & 0 & No obvious staining & 0 & $\mathrm{~S} \leq 1$ & Negative (-) \\
\hline $0<\mathrm{N} \leq 1 / 3$ & 1 & Pale yellow & 1 & $1<\mathrm{S} \leq 2$ & Weakly positive (+/-) \\
\hline $1 / 3<\mathrm{N} \leq 2 / 3$ & 2 & Brownish yellow & 2 & $2<\mathrm{S} \leq 4$ & Positive (+) \\
\hline $\mathrm{N}>2 / 3$ & 3 & Chocolate brown & 3 & $\mathrm{~S}>4$ & Strongly positive $(++)$ \\
\hline
\end{tabular}

VEGF, vascular endothelial growth factor; N, proportion of stained cells; S, score; ${ }^{\text {aS1xS2. }}$
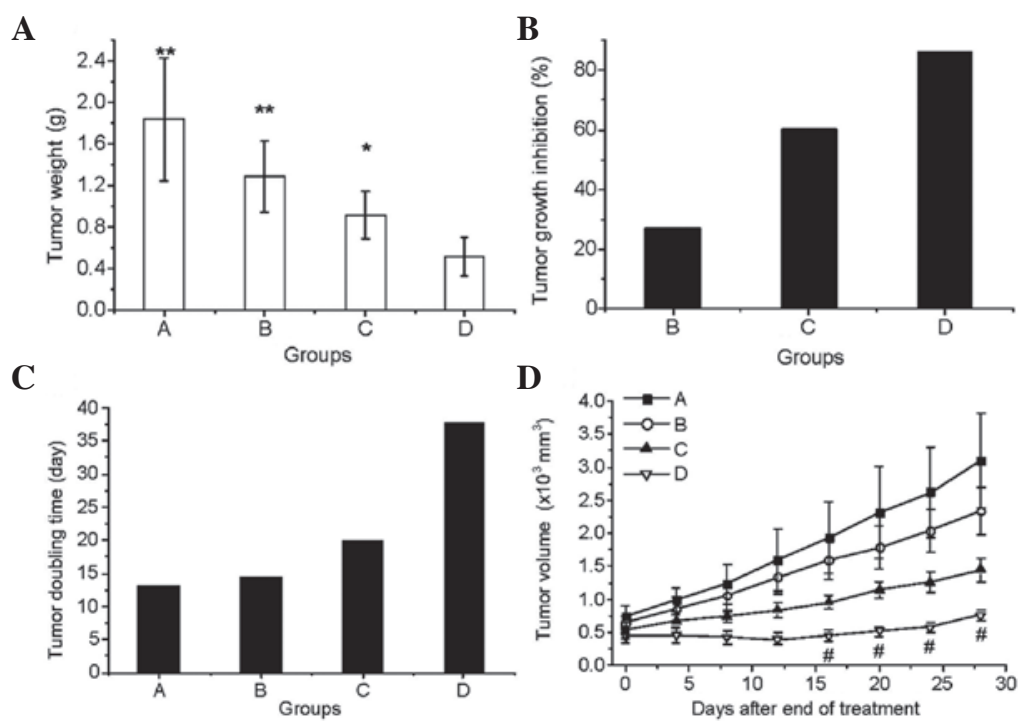

Figure 1. Therapeutic effects of the different treatments on NPC tumors in mice. Mice with NPC tumors were divided into four groups ( $\mathrm{n}=6$ ). Groups A, B, C and $\mathrm{D}$ were treated with normal saline, Endostar, radiation and Endostar + radiation, respectively. The tumor growth inhibition in group A was 0 ; this was not included in the graph. ${ }^{*} \mathrm{P}<0.05$ and ${ }^{* *} \mathrm{P}<0.01$ vs. group $\mathrm{D},{ }^{*} \mathrm{P}<0.05$ vs. group $\mathrm{C}$. NPC, nasopharyngeal carcinoma.

inhibition (Fig. 1B) and tumor doubling time (Fig. 1C) between the combination treatment group $\mathrm{D}$ and the other three groups $(\mathrm{P}<0.05$; Fig. 1$)$. The average tumor weights of the normal saline (A), Endostar (B), radiation (C) and Endostar + radiation (D) groups were 1.849 \pm 0.596 , $1.293 \pm 0.346,0.926 \pm 0.233$ and $0.523 \pm 0.180 \mathrm{~g}$, respectively (Fig. 1A). The tumor inhibition rates, calculated using the tumor volumes, in groups B, C and D were 27.12, 60.45 and $86.11 \%$, respectively (Fig. 1B). The tumor doubling time was also prolonged dramatically, particularly in the combined treatment group (Fig. 1C). The therapeutic effects on the growth of the tumor (Fig. 1D) showed that the tumor size in the Endostar + radiation group was significantly smaller than that of the other groups 16, 20,24 and 28 days after treatment (on days 26, 30, 34 and 38).

Effect of Endostar combined with radiation on MVD. The typical immunohistochemical staining images of CD34 and the effects of the various treatments on the MVD are shown in Fig. 2. These results indicate that Endostar or radiation monotherapy decreased the MVD, and the combination of Endostar and radiation significantly decreased the MVD of the tumor tissue in group $\mathrm{D}$ compared with that in group $\mathrm{A}(\mathrm{P}<0.01)$, group $\mathrm{B}(\mathrm{P}<0.05)$ and group $\mathrm{C}(\mathrm{P}<0.05)$.
Expressions of VEGF in NPC tumor tissues. The typical immunohistochemical staining images of VEGF are shown in Fig. 3. The results indicate that the VEGF expression in mice treated with normal saline, Endostar, radiation and Endostar + radiation were positive, weakly positive, strongly positive and weakly positive, respectively (Fig. 3).

\section{Discussion}

NPC animal models from nude mice are a viable research tool showing a high activity of NK cells, B cells and T cell-independent cells. The selection of nude mice for constructing animal models is appropriate due to their short experimental period, high tumor formation rate and good data comparability. The present study succeeded in the subcutaneous inoculation of the NPC cell line CNE into the right lower limb of nude mice, which developed into squamous cell carcinoma, suggesting the successful construction of an NPC animal model. The results of the animal model show that the combination of Endostar with radiation is more effective than monotherapy (either Endostar or radiation) in the treatment of NPC tumors.

Several studies have indicated that antiangiogenesis therapy combined with chemotherapy increases the local control rate and long-term survival rate $(10,11)$. Wang et al reported that 

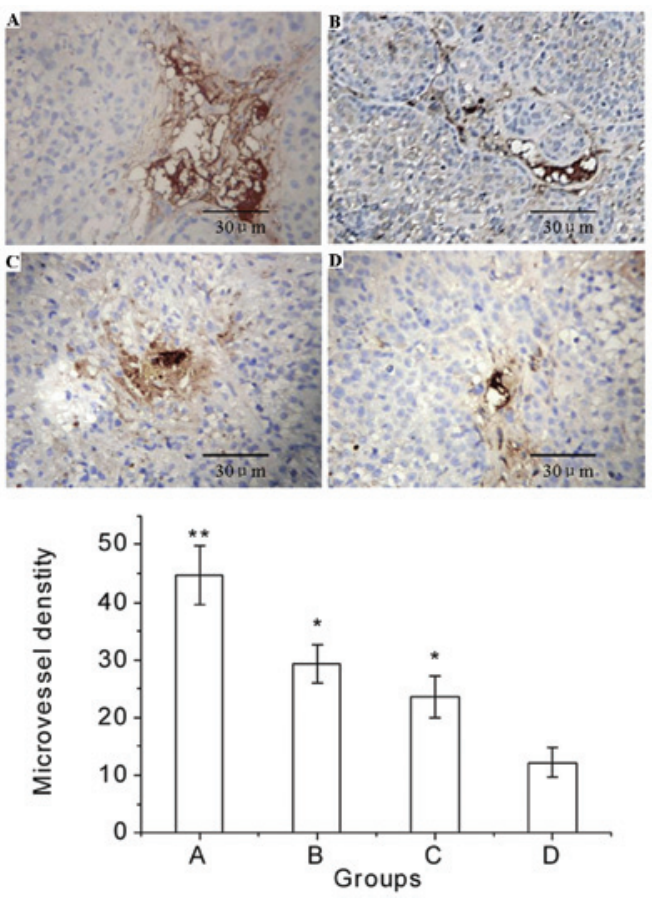

Figure 2. Hotspots of CD34 immunohistochemical staining and MVD in NPC tumor tissues. Tissues were treated with (A) normal saline; (B) Endostar; (C) radiation and (D) Endostar + radiation (x400). The histological sections stained for CD34 and developed with the DAB chromogen were examined to identify 5 separate zones (hotspots) containing high numbers of discrete CD34 staining sites (dyed claybank). The MVD of NPC tumor tissues in group $\mathrm{A}, \mathrm{B}$ and $\mathrm{C}$ were significantly different from group $\mathrm{D}\left({ }^{* *} \mathrm{P}<0.01\right.$ and ${ }^{*} \mathrm{P}<0.05$ vs. group D). MVD, microvessel density; NPC, nasopharyngeal carcinoma.

endostatin combined with adriamycin chemotherapy significantly controls liver cancer in mice (12). However, studies investigating the application of combined antiangiogenesis and radiation therapy are scarce. Several researchers believe that the inhibition of vascular formation decreases the blood supply to tumors and increases the ratio of hypoxic cells, resulting in a weakening of the effects of radiation. The results of the present study show that the combination of Endostar with radiation significantly inhibits the growth and metastasis of the NPC tumors inoculated with CNE1 cells, which is consistent with the studies by Teicher et al (13), Hansen-Algenstaedt et al (14), Luo et al (15) and Itasaka et al (16). In the present study, the sensitivity of NPC tumors to irradiation, recurrence time and local control rate were significantly increased in the combined treatment group that received Endostar for 10 days and were irradiated locally once on day 10 . These results suggest that the combination of local fractionated radiation with Endostar achieves better tumor control rates.

The present study also shows a decrease in MVD in the Endostar and combination groups compared with the other two groups $(\mathrm{P}<0.05)$. The present study suggests that Endostar improves tumor oxygenation (17) and sensitivity to radiation mainly by decreasing tumor microvessel density, depriving premature vessels of blood and increasing the blood flow to major vessels.

Radiation therapy controls the growth of tumor cells by killing them but it also induces an increase in VEGF expression in tumor cells. This increases tumor resistance to radiation and
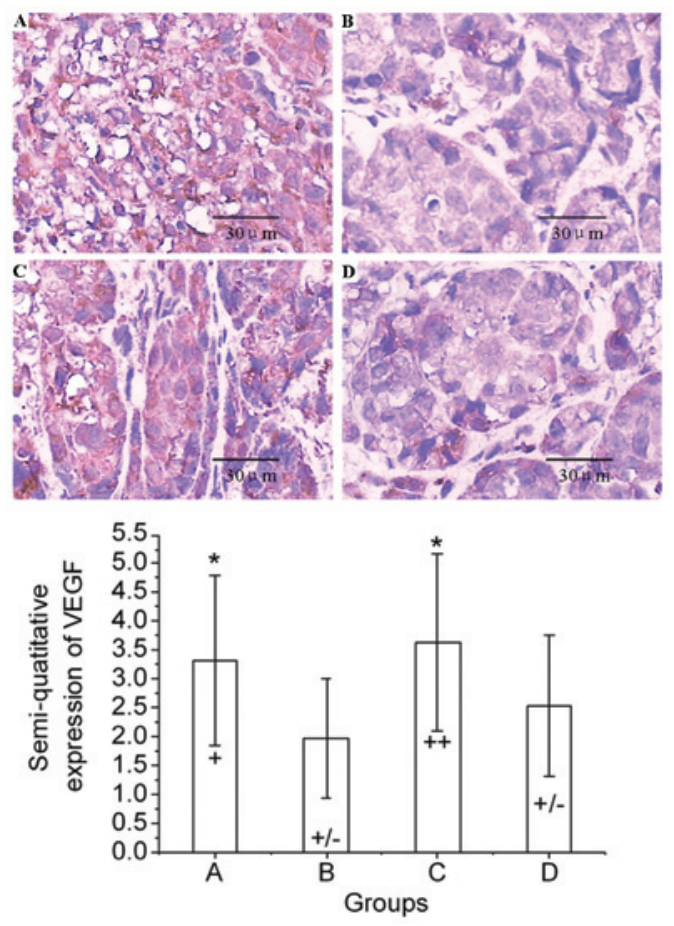

Figure 3. Immunohistochemical staining images of VEGF in tumor sections. Tissues were treated with (A) normal saline; (B) Endostar; (C) radiation and (D) Endostar + radiation $(n=6)(x 200)$. The VEGF expression in groups A, $\mathrm{B}, \mathrm{C}$ and $\mathrm{D}$ were evaluated as positive, weakly positive, strongly positive and weakly positive, respectively. Semi-quantitative VEGF expression levels in groups $\mathrm{A}$ and $\mathrm{C}$ were significantly different from group $\mathrm{D}(\mathrm{P}<0.05)$. VEGF, vascular endothelial growth factor.

promotes the formation of tumor vessels (18). It also increases their ability to invade other tissues by increasing the activity of matrix lyase in tumor cells (19). Therefore, radiation can protect tumors and decrease their sensitivity to further radiation therapy. Topolovec et al also verified that VEGF and MVD act as prognostic factors in endometrial cancer (20). The current study demonstrates that Endostar lowers VEGF expression in NPC tumors, which lowers the ability of vascular endothelial cells to repair radiation damage and increases the sensitivity of NPC tumor cells to radiation. Hence, the combination of Endostar with radiation has a synergistic effect on killing NPC tumors.

Combining Endostar with radiation inhibits the growth of NPC xenograft tumors and increases the sensitivity of tumor cells to radiation. This strategy provides a new clinical treatment method for NPC. However, angiogenesis is a complicated process that is influenced by many factors. Radiation therapy may be classified according to exposure time and intensity of radiation. The present study is only a preliminary exploration into combining Endostar with radiation for treating NPC. The underlying mechanisms and other alternatives require further study.

\section{Acknowledgements}

This study was supported by the Social Development Fund of Suzhou Science and Technology Bureau (grant number SYS201013). 


\section{References}

1. Yin WB and Gu XZ (eds): Nasopharyngeal carcinoma. In: Radiation Oncology. 4th edition. Peking Union Medical College Press, Beijing, p480, 2007.

2. Kwong D, Sham J and Choy D: The effect of loco-regional control on distant metastastic dissemination in nasopharyngeal carcinoma: an analysis of 1301 patients. Int J Radiat Oncol Biol Phys 30: 1029-1036, 1994.

3. Langeland Marthinsen AB, Dybdahl Wanderas A, Lundgren S, Strickert T, et al: Effects of growth factors on growth and radiation sensitivity of the human breast cancer cell line T-47D. Oncol Rep 9: 397-403, 2002.

4. Hovinga KE, Stalpers LJ, Van Bree C, et al: Radiation-enhanced vascular endothelial growth factor (VEGF) secretion in glioblastoma multiforme cell lines - a clue to radioresistance. J Neurooncol 74: 99-103, 2005.

5. Folkman J: Role of angiogenesis in tumor growth and metastasis. Semin Oncol 29: 15-18, 2002.

6. Feldman AL, Restifo NP, Alexander HR, et al: Antiangiogenic gene therapy of cancer utilizing a recombinant adenovirus to elevate systemic endostatin levels in mice. Cancer Res 60: 1503-1506, 2000.

7. Ling Y, Yang Y, Lu N, et al: Endostar, a novel recombinant human endostatin, exerts antiangiogenic effect via blocking VEGF-induced tyrosine phosphorylation of KDR/Flk-1 of endothelial cells. Biochem Biophys Res Commun 361: 79-84, 2007.

8. Mäkitie T, Summanen P, Tarkkanen A, et al: Microvascular density in predicting survival of patients with choroidal and ciliary body melanoma. Invest Ophthalmol Vis Sci 40: 2471-2480, 1999.

9. Tokuraku M, Sato H, Murakami S, et al: Activation of the precursor of gelatinase A/72 kDa type IV collagenase/MMP-2 in lung carcinomas correlates with the expression of membranetype matrix metalloproteinase (MT-MMP) and with lymph node metastasis. Int J Cancer 64: 355-359, 1995.

10. Gerger A, El-Khoueiry A, Zhang W, et al: Pharmacogenetic angiogenesis profiling for first-line Bevacizumab plus oxaliplatin-based chemotherapy in patients with metastatic colorectal cancer. Clin Cancer Res 17: 5783-5792, 2011.
11. Ma J, Chen CS, Blute T and Waxman DJ: Antiangiogenesis enhances intratumoral drug retention. Cancer Res 71: 2675-2685, 2011.

12. Wang ZX, Wang SM, Zhou Q, et al: Endostatin in different administration routes combined with adriamycin chemotherapy in the treatment of liver cancer xenograft in mice. Nan Fang Yi Ke Da Xue Xue Bao 30: 1903-1905, 2010 (In Chinese).

13. Teicher BA, Dupuis N, Kusomoto T, et al: Antiangiogenic agents can increase tumor oxygenation and response to radiation therapy. Rad Oncol Invest 2: 269-276, 1994.

14. Hansen-Algenstaedt N, Stoll BR, Padera TP, et al: Tumor oxygenation in hormone-dependent tumors during vascular endothelial growth factor receptor- 2 blockade, hormone ablation, and chemotherapy. Cancer Res 60: 4556-4560, 2000.

15. Luo X, Slater JM and Gridley DS: Radiation and endostatin gene therapy in a lung carcinoma model: pilot data on cells and cytokines that affect angiogenesis and immune status. Techol Cancer Res Treat 5: 135-146, 2006.

16. Itasaka S, Komaki R, Herbst RS, et al: Endostatin improves radioresponse and blocks tumor revascularization after radiation therapy for A431 xenograft in mice. Int J Radiat Oncol Biol Phys 67: 870-878, 2007.

17. Lee CG, Heijn M, di Tomaso E, et al: Anti-vascular endothelial growth factor treatment augments tumor radiation response under normoxic or hypoxic condition. Cancer Res 60: 5565-5570, 2000.

18. Abdollahi A, Lipson KE, Han X, et al: SU5416 and SU6668 attenuate the angiogenic effects of radiation-induced tumor cell growth factor production and amplify the direct antiendothelial action of radiation in vitro. Cancer Res 63: 3755-3763, 2003.

19. Kaliski A, Lassau L, Maggiorella L, et al: Antiangiogenic effect and tumor growth control achieved by an MMP-inhibitor combined to radiation in vivo, targeting radio-induced MMP-2 enhancement and VEGF modulation. Int $\mathrm{J}$ Radiat Oncol Biol Phys 60: 368-369, 2004.

20. Topolovec Z, Corusić A, Babić D, et al: Vascular endothelial growth factor and intratumoral microvessel density as prognostic factors in endometrial cancer. Coll Antropol 34: 447-453, 2010. 